\title{
LA INTERACTIVIDAD EN LA EDUCACION A DISTANCIA: EVALUACION DE COMUNIDADES DE APRENDIZAJE
}

\author{
(INTERACTIVITY IN DISTANCE EDUCATION: EVALUATION OF ONLINE LEARNING COMMUNI- \\ TIES)
}

Helga Stokes

State University Pennsylvania (EE.UU.)

\section{RESUMEN}

La tecnología de computadoras y la Internet permiten que haya una interactividad aumentada en la educación a distancia. Se crean así comunidades de aprendizaje en línea. Los estudiantes se involucran en un discurso y resulta aprendizaje construido de forma social.

El aprendizaje construido de manera social no se puede "empaquetar" dentro de objetivos bien definidos de aprendizaje. Es difícil medir si la conversación entre los estudiantes resulte, de verdad, en nuevas destrezas y conocimientos y de qué manera las características de la localidad virtual apoyen el proceso de aprendizaje.

El artículo explora el formato de unos espacios de aprendizaje a distancia en línea diseñados para el uso de los profesores. Se presentan posibles criterios de evaluación. Se discute la aplicabilidad de los criterios a la evaluación del formato de educación a distancia, el cual es tan flexible.

\begin{abstract}
Computer technology and the Internet allow for increased interactivity in distance education. Online learning communities are being created. Students are engaging in discourse, and socially constructed learning is taking place.

Socially constructed learning cannot be packaged in neatly defined learning goals. It is hard to measure whether the discourse amongst the learners truly generates new skills, insights, and knowledge and in what ways the features of the virtual site support the learning process.

The paper explores the format of selected online distance learning spaces for teachers, presents possible evaluation criteria, and discusses the applicability of the criteria to the evaluation of such a variable distance education format.
\end{abstract}




\section{LA INTERACTIVIDAD}

La interactividad como característica de los ambientes en línea ha abierto vías para el aprendizaje en colaboración, el establecimiento de comunidades profesionales de aprendizaje en línea, y el intercambio de información y opiniones. Tal intercambio es capaz de ser abierto, fluido, y de extender las fronteras de la instrucción planificada y presentada por expertos.

Tradicionalmente, la norma para la educación a distancia (DE, por sus siglas en inglés) ha sido la instrucción en base a la transmisión de conocimientos, diseñada y presentada por expertos. Sin embargo, en un ambiente en línea que apoye de verdad la comunicación, la adquisición de nuevos conocimientos y nuevas destrezas puede ser, en potencial, impulsada por el aprendiz. Las llamadas comunidades profesionales de aprendizaje utilizan aquellas posibilidades de la comunicación mediada por computadora para formar redes entre los practicantes profesionales, quienes comparten intereses y problemas comunes. Ante estas redes informales, para el intercambio de conocimientos y apoyo mutuo, únicamente existían a nivel espontáneo entre los colaboradores encontrados bajo un mismo techo. Con la disponibilidad actual de Internet, la distancia entre colaboradores presenta menos obstáculo.

\section{LA CONSTRUCCIÓN SOCIAL DE CONOCIMIENTO}

Quisiera enfocarme en la interactividad y el aprendizaje construido socialmente dentro del marco de desarrollo profesional de los maestros por medio de la educación a distancia, pues se ven dos tendencias convergentes las que hacen de esto un tema central.

Una es el cambio de práctica en el aula. Los conceptos pedagógicos a que se refieren frecuentemente son aquellos tales como "centrado en el aprendiz", "a base de investigación", "a base de proyecto", y "a base de problema". Estas son metodologías que provienen de un punto de vista constructivista y constructivista social con respecto al aprendizaje, lo que significa un ambiente en el cual el aprendiz construye nuevos conocimientos en interacción con el medio ambiente, incluyendo a los compañeros de clase (J onassen, 1999). Se espera que en tal ambiente los maestros implementen métodos correspondientes en sus aulas. Sin embargo, puesto que ellos proceden a menudo de la tradición magisterial de la exposición y el recuerdo de la información, cuentan con poca experiencia para llevarlo a cabo (Barab y otros, 2001). Dede (2004 a , p. 2), refiriéndose al contexto de los Estados Unidos observa: "Para preparar adecuadamente a los estudiantes para el trabajo y la ciudadanía del siglo XXI, el sistema de educación de los EE.UU. debe transformarse de modo que respalde el aprendizaje a base de investigación en la aula, en casa, y en la comunidad, puesto que esta es la manera por la cual se adquieren las destrezas complejas, tales como el pensar creativamentey la colaboración". 
La segunda tendencia es la popularidad creciente de las redes interactivas que apoyan el desarrollo profesional de los maestros. El aprendizaje a distancia se utiliza a menudo para cumplir con la demanda para el adiestramiento eficiente de un gran número de maestros, incluyendo a veces a aquellos ubicados en zonas lejanas. Por tradición, la radio, los materiales impresos y, donde esté disponible, la televisión han desempeñado el gran papel de satisfacer aquella necesidad. La UNESCO, por ejemplo, llevó a cabo diez estudios de caso acerca del uso de la educación a distancia para el adiestramiento de maestros (Kvaternik, 2001). Todos los casos estudiados utilizaron contenidos desarrollados por expertos y entregado a los maestros por medios impresos, radiofónicos, o la televisión. Sin embargo, los programas radiofónicos y los materiales impresos no se prestan bien para facilitar la comunicación entre los aprendices a través de la distancia. A menos que los centros de aprendizaje fueran establecidos en lugares donde los participantes pudieran reunirse en un ambiente en presencia, se halló que los aprendices no se comunicaron los unos con los otros. La utilización de las tecnologías de la comunicación (ICT, por sus siglas en inglés) cambio esta situación drásticamente, específicamente debido al uso del correo electrónico y los "kioskos electrónicos" (bulletin boards) para la comunicación asíncrona a base de computadora (CMC, pos sus siglas en inglés) o los "cuartos de conversación" (chat rooms) para la CMC síncrona.

Estas posibilidades para la interactividad asumieron gran importancia en el desarrollo profesional de maestros porque les permitió que emprendieran un papel activo en el diseño de sus propias experiencias de aprendizaje, o sea, en la práctica del aprendizaje por investigación, el mismo acercamiento educativo que se espera practiquen en sus aulas con sus propios alumnos. De esta manera, el medio vuelve a ser el mensaje. Si los maestros sólo absorbieran unos refranes acerca del aprendizaje por investigación, pero jamás lo aplicaran por si mismos, ¿cómo se espera que utilicen dicha práctica con sus estudiantes? “... los procesos del desarrollo profesional en turno a comunidades de aprendizaje reflejan el tipo de cambio que se desea en la práctica educativa - desde un movimiento de la asimilación pasiva de la información hacia la construcción activa del conocimiento, de tal modo que el proceso de innovar sea consistente con su contenido" (Dede, 2004ª, p.3).

Esta es precisamente la discrepancia que pueden remediar los rasgos interactivos de las distintas redes en línea que sirven para el desarrollo profesional de los maestros.

\section{Dos Ejemplos: “Tapped In" y "Red Escolar"}

Antes de hablar de la evaluación de la interactividad y las discusiones en línea, se proveen dos ejemplos escogidos de entre los muchos que sirven para ilustrar. El primero, Tapped In (SRI International, página de Web principal) que se desarrolla en los EE.UU. El segundo, Red Escolar, se radica en México (Red Escolar).

Taladrado ofrece varios rasgos comunicativos que se prestan favorablemente para establecer comunidades de aprendizaje profesional. El enfoque principal de Tapped In 
es el respaldo a una comunidad de aprendizaje profesional donde los participantes pueden encontrar información pertinente a su comunidad (Hoadley y Pea, 2002).

El concepto de Tapped In procede de aquel reconocimiento creciente que ninguna organización por si sola puede satisfacer el desarrollo continuo profesional de los maestros, que requiere que los educadores y los proveedores formen comunidades para compartir estrategias, recursos, y respaldo. Mediante Tapped In, los educadores piden extender su crecimiento profesional más allá de cursillos o talleres por medio de herramientas en línea, recursos, colegas y el apoyo que se necesite para poder implementar, de manera efectiva, actividades de aprendizaje que se enfoquen al estudiante.

- Los rasgos actuales de Tapped In son:

1. "Edificios" virtuales, hecho a medida, con "cuartos" (espacios) públicos, grupales, y personales.

2. Membresía con marbete de usuario y páginas de entrada [log-in].

3. Creación y gerencia por grupos.

4. Acceso para invitados y miembros por medio de los miradores de Web browsers comúnmente disponibles.

- Comunicación:

1. Conversación y mensajes privados en texto.

2. Sitios de discusión por tema disponibles en cada cuarto.

3. Transcripciones de conversaciones enviadas automáticamente por correo electrónico.

4. Mensajes guardados para aquellos que no estén en línea en algún momento.

- Compartir y recuperar información:

1. Almacenamiento de archivos y direcciones para localizar las páginas Web en los cuartos personales y grupales.

2. "Post-it" para mensajes de bienvenida, agendas, etc.

3. Directorios de usuarios temporales, grupos y miembros.

4. Búsqueda de miembros y otros recursos.

Tapped In puede ser utilizado tanto por los maestros para construir redes de aprendizaje con colegas, como por los entrenadores para hacer llegar sus cursillos. Como ya se explicó, se organiza en forma de "salas" que se dedican a distintas actividades de aprendizaje y cuentan con posibilidades para varias vías de comunicación.

La Red Escolar es un recurso para maestros en forma de sitio de Web. Se describe como un recurso que "ofrece las mismas oportunidades educativas a todos los mexicanos" (Red Escolar, página de Web principal). Los maestros tienen acceso al desarrollo profesional, materiales didácticos, y foros con propósito de intercambiar observaciones, experiencias, opiniones, y prácticas útiles. Este sitio complementa un plan para equipar a todas las escuelas primarias y secundarias con computadoras con conexión a Internet. 
El sitio ofrece espacio para proyectos de colaboración, adiestramiento y actividades permanentes, y provee acceso a una biblioteca y servicios educativos. Temas especiales, los que se refieren a las noticias de actualidad en la educación o señalan recursos interesantes, se destacan en la página principal.

Los foros son de interés especial. Algunos ejemplos de discusiones en línea pueden verse en el "Foro Enseñar a Enseñar", diseñado de tal forma que puede ser utilizado por los maestros de todas disciplinas, y en otro foro para los maestros en aulas con computadoras. Se pueden encontrar discusiones organizadas por temas en los dos. Los participantes someten mensajes relacionados a un tema u otro, reflexionan en torno a su práctica educativa, piden y dan consejos, intercambian experiencias, expresan opiniones y señalan otros recursos de utilidad. De esta manera, los maestros se apoyan los unos a los otros en su crecimiento profesional.

Ambos proyectos ofrecen oportunidades amplias a los maestros aprendices a que construyan conocimientos dentro del marco de cursos formales moderados, el intercambio libre y espacios de trabajo en colaboración para proyectos específicos. Se pueden hojear varias conversaciones por tema en los dos sitios.

\section{METODOLOGÍAS DE EVALUACIÓN}

Varios acercamientos se pueden tomar a fin de evaluar a una comunidad de aprendizaje en línea. Estos incluyen un cálculo del número de participantes o de los mensajes sometidos, una encuesta de la satisfacción de los participantes y un análisis del contenido de las interacciones (Guawardena y otros, 1997).

Un cálculo del número de participantes se realiza fácilmente, así estos están identificados en la mayoría de los casos y, por lo tanto, se sabe su número. Si pertenecen a un grupo definido con una asociación ya conocida, se puede establecer un porcentaje de participación.

Para evaluar los niveles de participación activa, se puede llevar a cabo un cálculo del número y diversidad de los mensajes sometidos a través de un período definido, y esto puede compararse con el número de participantes registrados. En algunas comunidades en línea, se manifiesta claramente quiénes enviaron mensajes, qué número se enviaron, y quiénes se registraron como usuarios en línea y no enviaron mensajes.

Algunos autores sugieren un análisis de flujo de mensajes en el cual se saca el total de los mensajes sometidos por segmentos de tiempo definidos durante una sesión. Estos investigadores hallaron que las conferencias comienzan de forma lenta, usualmente, demuestran un aumento de actividad, y entonces disminuye (Gunawardena y otros, 2001, p. 4). Puede que eso sea más aplicable a los intercambios síncronos, pues las comunicaciones asíncronas pueden extenderse por una duración de meses. Se han visto comunicaciones por tema que disminuyen, pero que se reestablecen después de un lapso de tiempo considerable. 
Una posible estrategia para evaluar los resultados de la efectividad de una sesión es el uso de los propios objetivos de aprendizaje (Inglis y otros, 2002, p. 178). Las comunidades de aprendizaje en línea (OLC, por sus siglas in inglés), se dan a conocer, a menudo, por sus temas y funciones dentro de una sección de objetivos y metas. Se puede utilizar tal información como estándar ("benchmark") y se puede tabular con referencia al mismo la frecuencia de los comentarios sometidos relacionados a cualquier tema. Los resultados revelan la importancia que los participantes asignen a un tema $u$ otro.

El análisis de la naturaleza de las interacciones dentro de una discusión en línea es el aspecto más complejo de la evaluación. Por lo tanto, enfoquémonos en ese aspecto.

\section{EVALUACIÓN DE LA INTERACTIVIDAD}

Se sugiere, según las normas para evaluar a una comunidad de aprendizaje en línea mediante análisis de las comunicaciones mediadas por computadora en los modos síncronos y asíncronos, que a fin de determinar si los participantes se beneficien del proceso, y desarrollen nuevos entendimientos y prácticas, se deben plantear una variedad de preguntas:

- ¿Qué tipo de actividad cognitiva ejecutan los participantes (p. ej., formular preguntas, aclarar, negociar, sintetizar, etc.)?

- ¿Cuáles son las clases de argumentos avanzados a través de las discusiones?

- ¿Cuáles son los recursos aportados por los participantes para apoyar la exploración de nuevas posibilidades (p. ej., la experiencia personal, referencias a la literatura, datos)?

- ¿Qué evidencia hay de cambios de entendimiento o la creación de una nueva construcción de conocimiento personal como resultado de las interacciones dentro del grupo?

(Gunawardena y otros, 1977, p. 412)

Varios investigadores han desarrollado maneras, gracias a las cuales se puede analizar la comunicación en un ambiente en línea. Entre ellos, debemos hablar de, Henri, Gunawardena, Lowe, Anderson, Carabajal, Garrison, y Archer ${ }^{1}$. Destaco aquí el Modelo de Análisis de la Interacción, desarrollado por Gunawardena y colegas, debido a que se enfoca precisamente en la evaluación de la construcción social del conocimiento. 


\section{Modelo de Análisis de la Interacción para Examinar la Construcción de Conocimiento Social a través de las Conferencias por Computadora}

Según nos informan Gunawardena y sus colegas, existen varias fases que caracterizan el proceso de la construcción social del conocimiento. Ellos desarrollaron un modelo de análisis de la interacción a fin de examinar este proceso en el contexto de la comunicación mediada por computadora (CMC, por sus siglas en inglés). El análisis se hace en torno a transcripciones de CMC. Se pueden utilizar transcripciones de las discusiones en línea acerca de varios temas sugeridos por el moderador. Estas se analizan con referencia a los procesos de aprendizaje, con la ayuda de dicho modelo, que precisa de las siguientes fases de interacción:

- Fase I: compartimiento y comparación de la información.

- Fase II: descubrimiento y exploración de la disonancia en la inconsistencia entre los conceptos, ideas o aseveraciones.

- Fase III: negociación o acuerdo sobre el significado y la construcción mutua de conocimiento.

- Fase IV: comprobación y modificación de la síntesis o la construcción propuesta.

- Fase V: declaración de acuerdo y aplicación del significado nuevamente construida.

(Gunawardena y otros, 1997, p. 414)²

Utilicé dicho marco para evaluar las discusiones por tema que tuvieron lugar dentro del ambiente de una comunidad de aprendizaje en línea diseñada para profesionales en la educación de adultos y la educación por extensión. Mi experiencia personal, habiendo sido la única persona a evaluar estas discusiones por tema, fue que la determinación de las categorías es muy subjetivo y algunas operaciones son más fáciles que otros para precisar. Por ejemplo, una de las operaciones es "una declaración de observación u opinión". Se supone que una observación sea una acción objetiva en la que algún suceso se observa mientras ocurre, mientras que una opinión es más o menos subjetiva. El hecho de que estas dos funciones se alistan juntas lo hace difícil asignarlas a los mensajes sometidos. Encontré que "hacer y contestar preguntas para clarificar algunos detalles de las aseveraciones" fue relativamente fácil de detectar, mientras que "comprobar con referencia a esquemas cognitivas existentes" parece ser difícil de detectar en las mismas. Si un solo evaluador llevara a cabo la categorización de los mensajes sometidos, la decisión acerca de cómo clasificar un comentario determinado sería algo subjetivo. No obstante, si varios evaluadores categorizarán cada mensaje, se podría establecer un nivel de confianza entre evaluadores y reducir la subjetividad (Rourke y otros, 2001, p. 7). Puede que la categorización revele tendencias, pero no represente una cuenta precisa del número de contribuciones por tema. Me parece que antes de ocupar tal instrumento, los evaluadores tendrían que hablar de sus percepciones de cada categoría y comprobarlas, utilizando una aseveración muestra, para poder lograr un cierto nivel de confianza investigativa entre ellos. 
De cualquier modo, según mi experiencia, puede que la aplicación de las categorías proporcione perspicacia acerca de las tendencias encontradas en cualquier intercambio, que ayude a determinar si una conversación está a nivel sólo de compartir información sencilla, o si genera alguna modificación de esquemas cognitivos. Quizás no sea posible categorizar con precisión cada contribución, pero emerge una impresión general de la naturaleza de un intercambio dado, y puede que ella vislumbre el carácter de los procesos cognitivos que ocurrieran. Gunawardena y colegas admiten que "puede que algunos elementos de los mensajes sean difíciles de asignar con certeza a una fase específica, disminuyendo así el nivel de confianza entre evaluadores" (Gunawardena y otros, 2001, p. 6).

Los mismos autores observaron también que "muchas, a lo mejor la mayor parte, de las conferencias [o sea, los intercambios de mensajes] no proceden más allá de la Fase II" (ibid., p. 4). Pude observar un fenómeno parecido en la evaluación que realicé.

La comunidad de aprendizaje en línea que evalué fue diseñada con propósito de intercambiar experiencias y conocimientos. Fue una comunidad de aprendizaje estructurada de forma libre, es decir, no formal. López Islas (2004), por otro lado, describe el uso del mismo instrumento para evaluar los cursos formales ofrecidos en línea por la Universidad Virtual (UV) del Instituto Tecnológico de Monterrey, México. Al final de la década de 1990, casi todos los miembros de la Facultad de la UV integraron alguna medida de colaboración en línea en sus cursos. Algunos utilizaron el aprendizaje distribuido en base a problemas (dPBL, por sus siglas en inglés). En tales casos, los estudiantes participaron en el aprendizaje socialmente construido y resolvieron problemas como elemento formal de la tarea del curso. La comunicación en línea tuvo propósitos bien definidos, fue sujeto a calificación docente, y fue respaldada por la naturaleza de la calidad de participación esperada.

López Islas y sus colegas evaluaron 1,078 mensajes escritos por estudiantes matriculados en dichos cursos, empleando el modelo de análisis de interacción (ibid., p. 306). Es de destacar que, resultó que también ellos clasificaron pocas contribuciones estudiantiles como pertenecientes a Fase IV (comprobar y modificar síntesis propuesta o co-construcción) o de Fase V (aseveraciones de acuerdo/aplicación de entendimiento nuevamente construido). Los investigadores hallaron considerable negociación de significado y co-construcción de conocimiento (Fase III), pero pocas expresiones de disonancia (Fase II).

¿Significa eso que las operaciones que reflejen destrezas más altas de conocimiento y la construcción mutua de conocimientos sean difícil de lograrse en un ambiente en línea? ¿O es que estas representan una destreza que pocos puedan dominar?, independientemente si se comuniquen en línea o cara a cara. ¿Puede que la destreza del moderador sea una influencia mayor, o, más bien, es la motivación y la preparación de los participantes el elemento clave? ¿O es que la naturaleza de la tarea a cumplirse dicte el contenido de los intercambios? Parece que si un resultado factible y concreto tiene que lograrse, entonces la comunicación descrita en las Fases II, III y IV debiera de ocurrir, mientras que las preguntas no conver- 
gentes hechas simplemente por amor a una buena discusión conducen a intercambios que son solamente expresión a observaciones y opiniones.

\section{Hacia el Aumento del Acercamiento de Evaluación}

Aquí quisiera proponer un segundo vistazo a la naturaleza de las tareas en que los estudiantes participan mientras se comunican en el ambiente de aprendizaje en línea. Como ya se ha dicho, el énfasis se pone en la construcción social de aprendizaje. Al momento, parece que se utiliza con frecuencia el dPBL. No obstante, puede que el aprendizaje en base a proyectos sea otro posible acercamiento. En esencia, los estudiantes se involucrarían en el diseño de una solución de problema o de un producto como culminación del proyecto. El aprendizaje tiene lugar mientras que los aprendices diseñan una entidad. Banathy (1991), define "diseño" como: "una indagación creativa, disciplinada, y orientada a decisión, que tiene el objetivo de:

a) Diagnosticar y describir la situación del problema de diseño.

b) Clarificar la razón para ocuparse en el diseño.

c) Establecer límites para la indagación al diseño.

d) Formular los valores, las ideas, y la imagen de un futuro sistema que servirá para guiar el diseño.

e) Definir expectativas, aspiraciones, propósitos, y requisitos para el sistema a diseñarse.

f) Crear y evaluar representaciones alternativas del futuro sistema.

g) Establecer criterios que sirvan para evaluar alternativas.

h) Por medio de dichos criterios, seleccionar la alternativa más prometedora.

i) Describir el futuro sistema.

j) Planificar el desarrollo del sistema, en base a su descripción” ( p 39).

Los pasos de diseño descritos por Banathy son operaciones en los que los aprendices se involucran mientras trabajan en un problema o proyecto, y estos pueden utilizarse como criterios para analizar la comunicación en línea entre los participantes que trabajen en tareas definidas. El término sistema puede interpretarse como el resultado o la solución del problema en el acercamiento del aprendizaje en base a problema, o como producto en aprendizaje en base al proyecto.

Si los problemas se asignan como parte de las tareas del curso y toman forma de simulaciones, puede que los aprendices no planifiquen para el desarrollo del sistema (paso J) y que el instructor, a su vez, prescriba algunos de los pasos iniciales, tales como el "diagnosticar y describir la situación del problema de diseño." Es mi experiencia, no obstante, que estos pasos ocurrirán, ya sea con algo de variación, en un ambiente de aprendizaje basado en un problema o en un proyecto.

Cuando los maestros construyen redes y colaboran en línea, como ilustran los ejemplos iniciales de redes de aprendizaje, es probable que ellos se involucren en tareas de diseño, ya sea a fin de revisar currículo, o para diseñar proyectos colaborativos con estudiantes en aulas dispersas, o comprobar métodos innovadores de 
enseñanza. El aprendizaje de los mismos maestros, si se basa en un paradigma de constructivismo social, debiera fijarse en tareas de la vida real creativas y orientadas al diseño.

\section{CONCLUSIÓN}

La evaluación de la comunicación en línea en el contexto de aprendizaje constructivista social, empleando operaciones que se hallan en la empresa de diseño, es una sugerencia que carece, en este momento, de mayor experimentación y comprobación. Estamos solamente al principio del uso de intercambios en línea para construir conocimientos, y las experiencias y evaluaciones formales todavía son pocos. Preguntas acerca del uso óptimo, ambientes que respalden, y otros temas por el estilo, están en su mayoría sin contestación.

Puesto que las escuelas requieren actualmente que los maestros utilicen métodos constructivistas y más centrados en el estudiante, es importante que los mismos maestros tengan experiencias propias con tales prácticas. Ya que la educación a distancia se utiliza como plataforma para el desarrollo profesional de los maestros y dado que la interactividad y la construcción social de conocimientos es posible ahora dentro del marco de la educación a distancia, las experiencias con estos acercamientos educativos debieran ser recogidas y examinadas de manera más amplia.

\section{NOTAS}

1. Ver Apéndice 1 por una tabla resumida de varios modelos.

2. Para el modelo completo con detalles de las operaciones, ver Apéndice 2. 


\section{Apéndice I \\ Modelos para la evaluación de una comunidad de aprendizaje en línea}

\begin{tabular}{|c|l|}
\hline Propósito de la Evaluación & \multicolumn{1}{|c|}{ Modelo de Evaluación } \\
\hline $\begin{array}{c}\text { Describir la naturaleza de las interac- } \\
\text { ciones entre aprendiz-aprendizy }\end{array}$ & $\begin{array}{l}\text { Henri (1992) } \\
\text { Rourke, Anderson, Garrison y Archer } \\
\text { aprendiz-maestro }\end{array}$ \\
$\begin{array}{l}\text { Gunawardena, Lowe y Anderson } \\
(1998)\end{array}$ \\
\hline $\begin{array}{c}\text { Examinar los procesos cognitivos } \\
\text { Analizar los roles del moderador y los } \\
\text { aprendices en línea }\end{array}$ & $\begin{array}{l}\text { Kirkley, Savery y Grabner-Hagen } \\
(1998)\end{array}$ \\
\hline
\end{tabular}

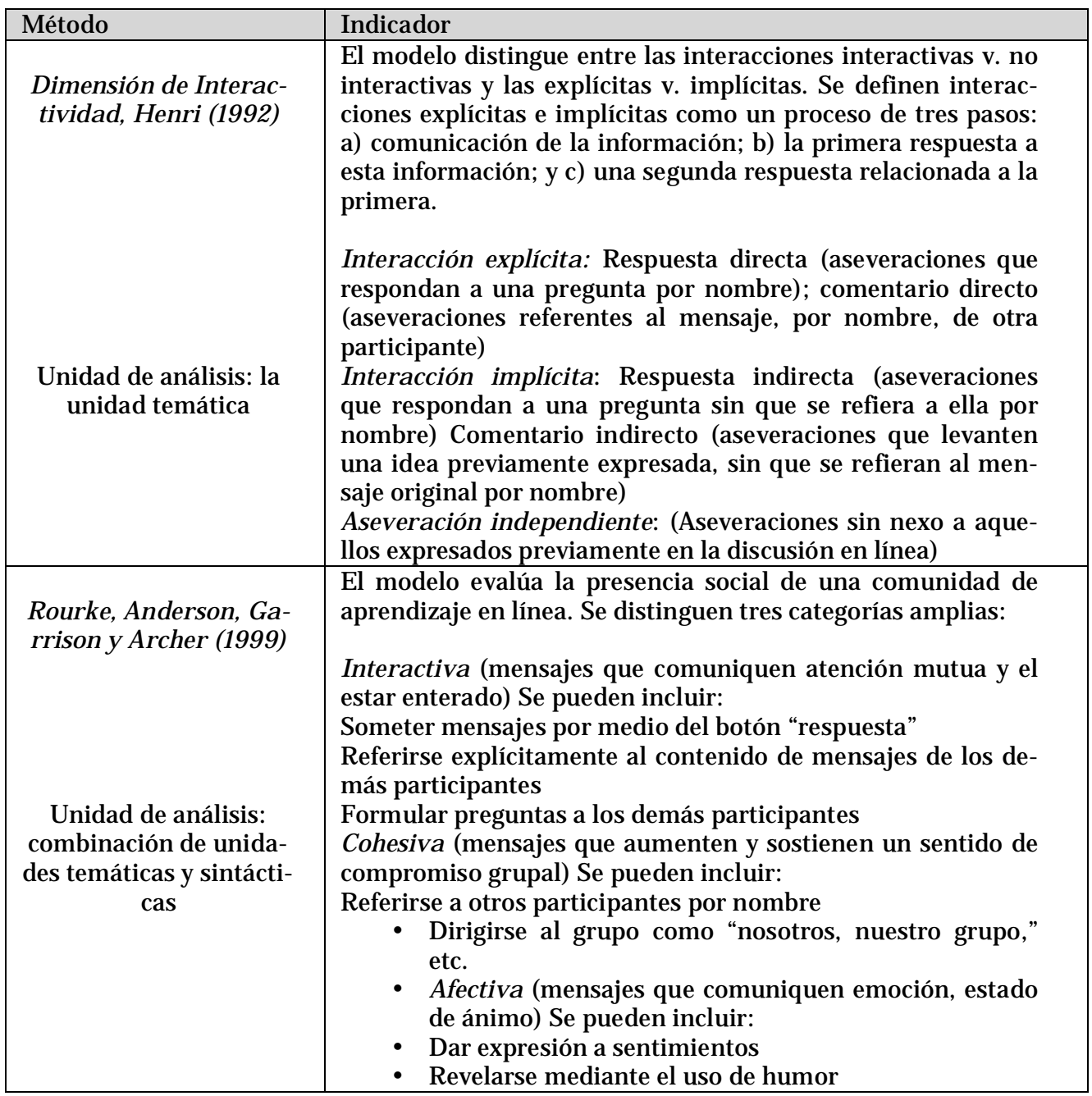




\begin{tabular}{|c|c|}
\hline Método & Indicador \\
\hline $\begin{array}{l}\text { Gunawardena, Lowey } \\
\text { Anderson (1997) }\end{array}$ & $\begin{array}{l}\text { El modelo evalúa la construcción social del conocimiento en } \\
\text { un foro de discusión en línea. Se identifican cinco fases en la } \\
\text { construcción de conocimiento: } \\
\text { Fase I: compartir/ comparar información, que puede incluir: } \\
\text { - aseveraciones de observación u opinión } \\
\text { - aseveraciones de acuerdo, provistos por uno o más de los } \\
\text { participantes } \\
\text { - ejemplos que concuerden, provistos por uno o más de los } \\
\text { participantes } \\
\text { - definición, descripción, o identificación del problema } \\
\text { Fase II: Descubrimiento de Disonancia, que puede incluir: } \\
\text { - identificación y declaración de áreas de desacuerdo } \\
\text { - plantear y contestar preguntas para clarificar la fuente y } \\
\text { el alcance del desacuerdo } \\
\text { Fase III: Negociación/ Construcción Mutua, que puede in- } \\
\text { cluir: } \\
\text { - negociación o clarificación del significado de términos } \\
\text { - identificación de áreas de acuerdo o coincidencia entre } \\
\text { los conceptos en conflicto } \\
\text { - Propuesta y negociación de nuevas aseveraciones que in- } \\
\text { corporen términos medios } \\
\text { Fase IV: Comprobación de construcciones tentativas, que } \\
\text { puede incluir: } \\
\text { - comprobación de la síntesis propuesta con referencia a } \\
\text { "hechos recibidos" compartidos por los participantes y/ o su } \\
\text { cultura } \\
\text { - comprobación con referencia a la experiencia personal } \\
\text { - comprobación con referencia a los datos recogidos for- } \\
\text { malmente } \\
\text { - comprobación con referencia al testimonio contradictorio } \\
\text { en la literatura } \\
\text { Fase V: Aseveración de Acuerdo, Aplicación de Significado } \\
\text { Construido de Nuevo, que puede incluir: } \\
\text { - resumen de acuerdo } \\
\text { - aplicación de nuevos conocimientos } \\
\text { - aseveraciones metacognitivas de parte de los participan- } \\
\text { tes, los que ilustren que se hayan cambiado su conocimiento } \\
\text { o modos de pensar (esquemas cognitivos) como resultado de } \\
\text { su interacción en línea }\end{array}$ \\
\hline $\begin{array}{c}\text { Dimensión Cognitiva } \\
\text { de Henri (1992) }\end{array}$ & $\begin{array}{l}\text { El modelo evalúa el pensamiento crítico de los aprendices en } \\
\text { línea } \\
\text { Pensamiento crítico. Hay cinco tipos distintos: } \\
\text { - Clarificación elemental - transmitir información sin } \\
\text { elaboración } \\
\text { - Clarificación profunda - analizar el problema, identi- } \\
\text { ficar asunciones } \\
\text { - Inferencia - concluir a base de evidencia de asevera- } \\
\text { ciones anteriores } \\
\text { - Juicio - expresar juicio acerca de una inferencia } \\
\text { - Estrategias - proponer una solución, bosquejar lo que } \\
\text { se necesite para implementarla }\end{array}$ \\
\hline
\end{tabular}




\begin{tabular}{|c|c|}
\hline Método & Indicador \\
\hline $\begin{array}{l}\text { Unidad de análisis: } \\
\text { unidad temática }\end{array}$ & $\begin{array}{l}\text { Cada uno de los cinco tipos de pensamiento crítico se clasifi- } \\
\text { can de acuerdo con la dicotomía de nivel de procesamiento de } \\
\text { información, de superficial a profundo } \\
\text { - Procesamiento superficial - repetir un mensaje sin } \\
\text { que se añada nueva información; aseverar sin justifi- } \\
\text { car; o sugerir solución sin explicación } \\
\text { - Procesamiento profundo - proporcionar nueva in- } \\
\text { formación, mostrar vínculos, proponer soluciones con } \\
\text { análisis de posibles consecuencias; proveer evidencia } \\
\text { de justificación }\end{array}$ \\
\hline $\begin{array}{l}\text { Newman, J ohnson, } \\
\text { Webb y Cochrane } \\
\text { (1997) }\end{array}$ & $\begin{array}{l}\text { El modelo mide el nivel de pensamiento crítico partiendo del } \\
\text { modelo de Henri (1992) ampliado. Se incluyen diez indicado- } \\
\text { res: } \\
\text { - relevancia } \\
\text { - importancia } \\
\text { - novedad } \\
\text { - el aportar conocimiento o experiencia desde afuera } \\
\text { - justificación } \\
\text { - evaluación crítica } \\
\text { - el vincular ideas o interpretarlas } \\
\text { - ambigüedad y claridad } \\
\text { - utilidad práctica } \\
\text { - amplitud de conocimiento } \\
\text { Cada uno de estos diez indicadores tiene su propio listado de } \\
\text { pares opuestos, uno que corresponde al nivel de procesa- } \\
\text { miento superficial, otro al profundo. P. ej., "aseveraciones } \\
\text { impertinentes o disparates" v. "aseveraciones relevantes". }\end{array}$ \\
\hline $\begin{array}{l}\text { Unidad de análisis: } \\
\text { Contenido instructivo } \\
\text { de cada oración, una } \\
\text { por una }\end{array}$ & $\begin{array}{l}\text { El modelo evalúa los distintos modos de ayuda al aprendizaje } \\
\text { que un moderador en línea pueda ofrecer a los aprendices. } \\
\text { - Andamiaje - se refiere al apoyo, guía y comentarios } \\
\text { que se ofrecen al aprendiz a fin de que domine los } \\
\text { materiales y se mueva a un nivel de entendimiento } \\
\text { más alto. } \\
\text { - Retroalimentación acerca de la ejecución - informa- } \\
\text { ción, positiva o negativa, proporcionada por el mode- } \\
\text { rador o instructor acerca de actos o ideas específicos. } \\
\text { - Estructuración cognitiva - forma de ayuda que el } \\
\text { moderador/instructor para proveer una estructura } \\
\text { para pensar y actuar de modo que facilite al aprendiz } \\
\text { en línea a que organice la experiencia "cruda". } \\
\text { Modelación - ocurre cuando el moderador/instructor } \\
\text { actúa de forma que puede imitarse. } \\
\text { Manejo de contingencias - Utilizado para recompen- } \\
\text { sar el comportamiento mediante alabanza o aliento, o } \\
\text { controlar el comportamiento indeseable por medio de } \\
\text { castigo en forma de censura. } \\
\text { Instrucción - ocurre cuando el moderador/instructor } \\
\text { da información explícita en relación a actos específi- } \\
\text { cos. }\end{array}$ \\
\hline
\end{tabular}




\begin{tabular}{|l|l|}
\hline Método & Indicador \\
\hline & $\begin{array}{l}\text { Interrogación - se utiliza como impulsor para estimu- } \\
\text { lar pensamiento y provocar actividades creativas en el } \\
\text { aprendiz. }\end{array}$ \\
\hline
\end{tabular}

(Hew y Cheung, 2003)

\section{Anexo 2: Modelo de Análisis de la Interacción}

- Fase I: Compartir/Comparar la Información

Las operaciones en esta etapa incluyen:
A. Aseveraciones de observación u opinión
B. Aseveraciones de acuerdo de parte de uno o más de los participantes
C. Ejemplos que acuerden provistos por uno o más de los participantes
D. Plantear y contestar preguntas para clarificar detalles de las aseveraciones
E. Definición, descripción o identificación del problema

- Fase II: Descubrimiento y Exploración de Disonancia de las Inconsistencias entre Ideas, Conceptos o Aseveraciones

Las operaciones en esta etapa incluyen (alistadas selectivamente):
A. Identificación y declaración de las áreas de desacuerdo
B. Plantear y contestar preguntas para clarificar la fuente y el alcance del des- acuerdo
C. Reaseverar la posición del participante y, posiblemente, proponer argumen- tos o consideraciones que la respalden por medio de referencias a la expe- riencia del participante, a la literatura, a los datos formales recogidos, o me- diante planteamiento de una metáfora o analogía relevante para ilustrar un punto de vista

- Fase III. Negociación del Significado y Construcción Mutua de Conocimiento Las operaciones en esta etapa incluyen:
A. Negociación o clarificación del significado de términos
B. Negociación del peso relativo a asignarse a las clases de argumentos
C. Identificación de áreas de acuerdo o coincidencia de entre los conceptos que choquen
D. Propuesta y negociación de aseveraciones nuevas, que incorporen términos medios y/ o construcción mutua
E. Propuesta para integrar o acomodar metáforas o analogías

- Fase IV: Comprobación y Modificación de la Síntesis o Construcción Propuesta Las operaciones en esta etapa incluyen:
A. Comprobación de la síntesis propuesta con referencia a "hechos recibidos" compartidos entre los participantes y/ o su cultura
B. Comprobación con referencia a esquemas cognitivos ya existentes
C. Comprobación con referencia a la experiencia personal
D. Comprobación con referencia a los datos formales recogidos
E. Comprobación con referencia al testimonio contradictorio en la literatura

- Fase V: Aseveración de Acuerdo y Aplicación del Conocimiento Construido de Nuevo 
Las operaciones en esta etapa incluyen:
A. Resumen de los acuerdos
B. Aplicación de nuevos conocimientos
C. Aseveraciones metacognitivas de parte de los participantes que ilustren que se hayan cambiado su conocimiento o modos de pensar (esquemas cogniti- vos) como resultado de su participación en la interacción en línea

(Gunawardena, Lowe y Anderson, 1997)

\section{REFERENCIAS BIBLIOGRÁFICAS}

Banathy, B.H. (1991). Systems design of education: A journey to create the future. Englewood Cliffs, NJ : Educational Technology Publications

Barab, S. A.; MaKinster, J. G.; Moore, J. A; Cunningham, D. J.; The ILF Design Team, (2001). Designing and Building an On-line Community: The Struggle to Support Sociability in the Inquiry learning Forum. ETR\&D 49(4), 71-98

Dede, C. (2004a). Enabling Distributed Learning Communities via Emerging Technologies - Part One. T.H.E. J ournal Online. Disponible en: http:// www.thejournal.com/magazine/v ault/articleprintversion.cfm?aid=4963 [consulta 2005, 15 de septiembre]

Dede, C. (2004b). Enabling Distributed Learning Communities via Emerging Technologies - Part Two. T.H.E. J ournal, 32(3)

Gunawardena, C.N.; Lowe, C.A.; Anderson T. (1997). Analysis of a global online debate and the development of an interaction analysis model for examining social construction of knowledge in computer conferencing. Journal of Educational Computing Research, 17 (4). 395-429

Gunawardena, C.N.; Lowe, C.A.; Anderson T. (1998). Transcript Analysis of Computer-Mediated Conferences as a Tool for Testing Constructivist and SocialConstructivist learning Theories. ERIC Document Reproduction Service No. ED 422854
Gunawardena, C.N.; Carabajal, K; Lowe, C. (2001). Critical analysis of models and methods used to evaluate online learning networks. Seattle: American Educational Research Association. (ERIC Document Reproduction Service No. ED456159)

Hew, K. F.; Cheung, W. S. (2003). Models to evaluate online learning communities of asynchronous discussion forums. Australian J ournal of Educational Technology, 19(2), 241-259.

Hoadley, C. M.; Pea, R.D. (2002). Finding the ties that bind: Tools in support of a knowledge-building community. En: K. A. Renninger. K.A.; Shumar, W. (Eds.), Building virtual communities: Learning and change in cyberspace. New York: Cambridge University Press.

Inglis, A; Ling, P.; J oosten, V. (2002). Delivering Digitally: Managing the Transition to the Knowledge Media. London: Taylor \& Francis

Jonassen, D. (1999). Designing constructivist learning environments. In: Reigeluth, C., Instructional design theories and models: A new paradigm of instructional theory: VolumeII. Mahwah, NJ : Erlbaum, 215-239

Kvaternik, R., (2002). Teacher Education through Distance Learning: Technology, Curriculum, Evaluation, Cost. Paris: UNESCO

López-Islas J.R. (2004). Collaborative Learning at Monterrey Tech-Virtual Uni- 
versity. In: Duffy, T.M.; Kirkley, J.R. Learner-Centered Theory and Practice in Distance Education. Mahwah, NJ: Erlbaum, 297-320.

Red Escolar (México). Pagina de Web Principal. Disponible en: http://redescolar.ilce.edu.mx/ [consulta 2005, 17 de septiembre]

Rourke, L.; Anderson, T.; Garrison, D. R.; Archer, W. (2001). Methodological issues in the content analysis of computer conference transcripts. International Journal of Artificial Intelligence in Education 12

SRI International, Center for Technology in Learning. Tapped In. Disponible en: http://ti2.sri.com/tappedin/web/about.j sp [consulta 2005, 15 de septiembre]

\section{PALABRAS CLAVE}

Interactividad, comunidades de aprendizaje, evaluación.

\section{KEY WORDS}

Interactivity, communities of practice, evaluation.

\section{PERFIL ACADÉMICO DE LA AUTORA}

Helga Stokes, candidata al doctorado y asistente de investigación, Departamento de Educación Continua, Universidad Estatal de Pennsylvania, EE.UU.

Maestra escolar, doctorando en Sistemas de Instrucción y en Educación Comparada e Internacional. Tiene interés principal en el diseño de innovaciones y cambios realizados por los mismos usuarios en el entorno escolar y también en el uso de redes escolares en línea para apoyar dicha empresa.

Dirección postal: 712 West Beaver Ave. \#E State College, PA 16801 EE.UU. E-mail: hss117@psu.edu

Fecha recepción del artículo: 27. 03. 2006

Fecha aceptación del artículo: 07. 04. 2006 